\title{
Travelling waves in hybrid chemotaxis models
}

\author{
Benjamin Franz - Chuan Xue - Kevin J. \\ Painter • Radek Erban
}

Preprint version: April 3, 2018

\begin{abstract}
Hybrid models of chemotaxis combine agent-based models of cells with partial differential equation models of extracellular chemical signals. In this paper, travelling wave properties of hybrid models of bacterial chemotaxis are investigated. Bacteria are modelled using an agent-based (individualbased) approach with internal dynamics describing signal transduction. In addition to the chemotactic behaviour of the bacteria, the individual-based model also includes cell proliferation and death. Cells consume the extracellular nutrient field (chemoattractant) which is modelled using a partial differential equation. Mesoscopic and macroscopic equations representing the behaviour of the hybrid model are derived and the existence of travelling wave solutions for these models is established. It is shown that cell proliferation is necessary for the existence of non-transient (stationary) travelling waves in hybrid models. Additionally, a numerical comparison between the wave speeds of the continuum models and the hybrid models shows good agreement in the case of weak chemotaxis and qualitative agreement for the strong chemotaxis case. In the case of slow cell adaptation, we detect oscillating behaviour of the wave, which cannot be explained by mean-field approximations.
\end{abstract}

Keywords hybrid model · travelling wave $\cdot$ bacterial chemotaxis

Benjamin Franz $\cdot$ Radek Erban

Mathematical Institute, University of Oxford

24-29 St. Giles', Oxford, OX1 3LB, United Kingdom

E-mail: franz@maths.ox.ac.uk, erban@maths.ox.ac.uk

Chuan Xue

Department of Mathematics, Ohio State University 231 West 18th Avenue, Columbus, OH 43210, USA

E-mail: cxue@math.osu.edu

Kevin J. Painter

Department of Mathematics, Heriot-Watt University

Edinburgh, EH14 4AS, United Kingdom

E-mail: K.J.Painter@ma.hw.ac.uk 


\section{Introduction}

The wavelike spread of cell populations plays a fundamental role in many biological processes, including development 24, wound healing 38] and tumour invasion [16]. Bacterial populations show similar phenomena, with the pioneering studies of Adler 1 confirming the capacity of an E. coli population to form travelling bands via chemotaxis to extracellular signals. Mathematically, the extent to which chemotaxis can generate and sustain stationary travelling bands has motivated a number of studies, including the Keller-Segel model of Adler's experiments which is written in the form of coupled partial differential equations (PDEs) [20]. This early model necessitated a biologically unrealistic singularity in the chemotactic sensitivity to generate stationary travelling waves: a requirement that allows bacteria behind the wave to acquire infinite speeds and to avoid "dropping-out", an effect that leads to gradual dispersal of the band 40,15 .

This singularity requirement can be circumvented by incorporating other processes. The well known Fisher's equation 14 demonstrates travelling waves in systems coupling diffusion with logistic growth terms 14. Parabolic chemotaxis models with non-singular sensitivities but incorporating either logistic [22, 23, 30, or non-logistic [21,36, growth terms also admit travelling wave solutions. Other studies have shown that introduction of more complex nutrient terms can give rise to travelling waves, even when growth is absent 34,35. An experimental system which also included two chemicals - a chemoattractant and a nutrient source - was presented in 6, 7, with stationary or transient travelling waves obtained according to $t$ he formulation of the model [5,40]. Travelling waves in chemotaxis models have also been recently studied in [26, 25]; we also note the articles [19] and [37] for a review and analysis of travelling waves in PDE-based models. A comparison between mesoscopic (hyperbolic) and macroscopic (parabolic) PDEs has been presented in [27.

Relatively little exploration has been conducted into travelling wave formation for chemotactic models extending beyond PDE systems, in particular those introducing terms to account for the inherent noise of biological systems. One exception is the study of 9 , in which a multiplicative noise term was introduced into the Keller-Segel model and the existence of travelling waves has been demonstrated within this setting. Hybrid models, in which an individualbased model for bacterial behaviour is coupled to a continuum description of extracellular signals, naturally introduce stochastic effects and will be the focus of the present paper. Such a hybrid model was formulated in [15] where it was shown that under finite cell speeds only transient travelling waves formed, even with singular chemotactic sensitivity. The individual-based model was formulated in terms of the velocity-jump model with internal dynamics [12, 13, 41, and, in this paper, we extend the model in 15 to incorporate proliferation and death of bacteria. We analyse this system numerically and analytically with respect to its travelling wave properties, employing the biologically inspired chemotactic sensitivity presented in [40] and a linear growth term. We 
show that stationary travelling waves can be observed even in the absence of chemotaxis, although wave speeds are substantially increased in its presence.

The organisation of the paper is as follows: the full hybrid model is presented in Section 2 along with illustrative simulation results, while the corresponding continuum equations are derived under certain assumptions in Section 3, in Section 4 these continuum equations are analysed with respect to travelling wave properties; in Section 5 where a computational analysis and comparison of the models is presented; finally, we discuss our observations in Section 6

\section{Hybrid model of bacterial chemotaxis}

In this section we formulate the hybrid model of bacterial chemotaxis which will be investigated in this paper. The model is motivated by the behaviour of the bacterium E.coli and, in its most general form, includes cell movement, sensing and response to a chemical signal, consumption of the chemoattractant, cell proliferation and death. However, for analytical tractability, we will also explore simplified hybrid models which exclude some of these processes. Bacteria are modelled as agents with internal dynamics that represent the signal processing and response of each individual while the extracellular chemical is modelled using a PDE to describe its spatio-temporal concentration. The mathematical framework and simulation techniques are reviewed in [15]. We consider the model in an effectively one-dimensional domain representing a long but narrow tube, similar to the experimental set up considered in [1].

The motion of $E$. coli bacteria is controlled through the coordinated rotation of flagella distributed over the cell surface 2]. Counterclockwise rotation generates a propulsive bundle that results in straight line motion of the bacterium - a so-called "run" 3. Alternatively, clockwise rotation results in the outward flaying of flagella and a "tumble" - rotation with insignificant displacement. At the end of each tumble the bacterium chooses a new direction of movement, seemingly at random, and returns to the run phase. The lengths of the individual phases are independent from each other and distributed exponentially, yet they can be influenced by internal dynamics 2 .

Internal dynamics of the $E$. coli bacteria possess two principal features [4]: a quick excitation phase followed by slower adaptation. Specifically, changes in the extracellular signal concentration lead to quick excitation of the internal metabolism, signified through altered chemical concentrations inside the cell. Following excitation the internal concentrations revert slowly to normal in an adaptation process, even when the external signal remains at the raised level.

\subsection{Velocity jump model with internal dynamics}

Run-and-tumble dynamics are aptly modelled as a velocity-jump process 31 , 12. We denote by $N_{a}(t)$ the number of bacteria (agents) in the system at time 
$t$. The current state of the $i$-th individual, $i=1,2, \ldots, N_{a}(t)$, will be described using its position $x_{i} \in \mathbb{R}$, its velocity $v_{i}= \pm s \in \mathbb{R}$ and a set of internal state variables $\mathbf{y}_{i} \in \mathbb{R}^{m}$ that represent the states of components in the intracellular signal transduction network.

Here we concentrate on a cartoon version of the internal dynamics of bacteria written in terms of two internal variables 32,12 , i.e $m=2$. Internal variables $y^{(1)}$ and $y^{(2)}$ are governed by the equations

$$
\begin{aligned}
& \frac{\mathrm{d} y^{(1)}}{\mathrm{d} t}=\frac{S(x(t), t)-y^{(1)}-y^{(2)}}{t_{e}}, \\
& \frac{\mathrm{d} y^{(2)}}{\mathrm{d} t}=\frac{S(x(t), t)-y^{(2)}}{t_{a}},
\end{aligned}
$$

where $t_{e}$ is the excitation time, $t_{a}$ is the adaptation time, $t_{e} \ll t_{a}$ and $S(x(t), t)$ is the concentration of chemoattractant at the position of the bacterium $x(t)$ at time $t$. Furthermore, bacteria move with the velocity $v_{i}= \pm s$ governed through a velocity jump process with a turning frequency $\lambda=\lambda(\mathbf{y})$ that depends on the internal dynamics. In this paper, we will use the biologically motivated nonlinear turning kernel developed in [40. Hence, the full model of one individual over (a small) time step $\Delta t$ can be written as:

$$
\begin{aligned}
& x(t+\Delta t)=x(t)+v(t) \Delta t, \\
& v(t+\Delta t)=\left\{\begin{array}{c}
-v(t), \text { with probability } \lambda(\mathbf{y}(t)) \Delta t, \\
v(t), \text { otherwise, }
\end{array}\right. \\
& \lambda(\mathbf{y}(t))=\lambda_{0}\left(1-\frac{y^{(1)}(t)}{\kappa+\left|y^{(1)}(t)\right|}\right), \\
& y^{(1)}(t+\Delta t)=y^{(1)}(t)+\frac{S(x(t), t)-y^{(1)}(t)-y^{(2)}(t)}{t_{e}} \Delta t, \\
& y^{(2)}(t+\Delta t)=y^{(2)}(t)+\frac{S(x(t), t)-y^{(2)}(t)}{t_{a}} \Delta t,
\end{aligned}
$$

where $\lambda_{0}$ and $\kappa$ are positive constants.

In addition to the behaviour of an individual bacterium we define a signaldependent proliferation function $h(S): \mathbb{R}^{+} \mapsto \mathbb{R}$. We thereby interpret a positive value of $h(S)$ as a proliferation rate, meaning that in the infinitesimal interval $[t, t+\Delta t)$ a bacterium at position $x$ generates an exact copy of itself with probability $h(S(x(t), t)) \Delta t$. Similarly, a negative value of $h(S)$ means that the bacterium disappears (dies) with the probability $-h(S(x(t), t)) \Delta t$. In this paper, we will use the following form for the proliferation rate $h(S)$ :

$$
h(S)=\alpha\left(S-S_{c}\right),
$$

where $\alpha$ and $S_{c}$ are positive constants. 
2.2 Evolution of the extracellular chemoattractant

For the extracellular signal $S(x, t)$ we formulate a PDE that incorporates diffusion (with diffusion constant $D_{S} \geq 0$ ) and signal consumption by bacteria, the latter with signal dependent rate $k(S): \mathbb{R}^{+} \rightarrow \mathbb{R}^{+}$. The equation for $S$ therefore takes the form

$$
\frac{\partial S}{\partial t}=D_{S} \frac{\partial^{2} S}{\partial x^{2}}-k(S) \sum_{i=1}^{N_{a}(t)} \delta\left(x-x_{i}(t)\right) .
$$

For the remainder of the paper we employ a linear form for the consumption function $k(S)$ :

$$
k(S)=\beta S,
$$

where $\beta$ is a positive constant.

\subsection{Illustrative example}

The hybrid model framework presented in Sections 2.1 and 2.2 includes essential features of the more complicated hybrid chemotaxis models formulated in 10,39 . In this section we numerically show that these processes can give rise to travelling waves. For the numerical simulation we employ techniques described in [15]. In particular, for the extracellular signal $S(x, t)$, this means that the simulation is performed on the one-dimensional domain $[0, L]$ with initial condition $S(x, 0)=S_{\infty}>0$ and zero-flux boundary conditions. We consider $M+1$ regularly spaced grid points $r_{j}=j \Delta x, j=0, \ldots, M$, where $\Delta x=L / M$ and the values of $S\left(x_{i}, t\right)$ are advanced by a small time step $\Delta t$ and a forward Euler update rule:

$$
\begin{aligned}
S\left(r_{j}, t+\Delta t\right) & =S\left(r_{j}, t\right)+D_{S} \Delta t \frac{S\left(r_{j-1}, t\right)+S\left(r_{j+1}, t\right)-2 S\left(r_{j}, t\right)}{(\Delta x)^{2}} \\
& -k\left(S\left(r_{j}, t\right)\right) \Delta t \sum_{i=1}^{N_{a}(t)} K\left(r_{j}-x_{i}(t)\right) .
\end{aligned}
$$

In the above $K: \mathbb{R} \rightarrow \mathbb{R}^{+}$is the symmetric, normalised and non-negative kernel

$$
K(\xi)=\frac{1}{\sqrt{2 \pi \sigma^{2}}} \exp \left[-\frac{\xi^{2}}{2 \sigma^{2}}\right],
$$

where the kernel width $\sigma$ is a positive real number. Here, $K\left(r_{j}-x_{i}\right)$ represents the influence a bacterium at position $x_{i}$ has on grid point $j$.

The simulation of the individual bacterium is given in the full system (2.2)(2.6) and complemented by the birth and death processes described in Section 2.1. where we use the same time step $\Delta t$ as in (2.10). To calculate the necessary off-grid values of extracellular signal, we linearly interpolate from the 
two nearest grid points. We further simplify the system (2.2) -2.6) by exploiting the separate time scales for excitation and adaptation (i.e. $t_{e} \ll t_{a}$ ): specifically, we assume the update equation (2.5) for $y^{(1)}$ is in a quasi-equilibrium, which is identical to the assumption $t_{e}=0$. The value for $y^{(1)}$ can therefore be calculated by

$$
y^{(1)}(t)=S(x(t), t)-y^{(2)}(t) .
$$

Illustrative results are presented in Figure 1 . For this simulation, $N_{a}(0)=10^{4}$ bacteria were initialised at positions $x_{i}(0)$, randomly generated as the absolute value of a Gaussian random variable with variance much smaller than the domain length $L$. The initial velocity (direction of movement) is generated uniformly at random and initial values of the extracellular signal and internal variables are taken as

$$
\begin{aligned}
y_{i}^{(1)}(0)=S_{\infty}, y_{i}^{(2)}(0)=0, & \text { for } i=1,2, \ldots, N_{a}(0), \\
S(x, 0) \equiv S_{\infty} & \text { for } x \in[0, L],
\end{aligned}
$$

where $S_{\infty}=1$. We simulate the system until time $T_{\text {final }}=100$ and plot both the distribution of bacteria and concentration of chemoattractant $S$ in Figure 1(a). We also estimate the wave speed as a function of time in Figure 1(b). We clearly see formation of a travelling band of bacteria, moving rightwards with average speed $v=0.51$ (plotted as the dashed line in Figure 1(b)).

\section{Influence of the growth term}

To investigate the influence of the growth term on the existence of travelling waves, we simulate the full hybrid model (2.2)-(2.6) and (2.10) including $(\alpha=1)$ and excluding $(\alpha=0)$ growth and death processes. We use identical parameters to those described above and present the results in Figure 2 In Figure 2(a) the position of the wave front (defined as the right-most position for which $S(x)<0.9$ ) is compared. The full hybrid system (dashed line) generates a straight line, indicating a wave moving with constant speed. While the system excluding growth and death (solid line) moves with a similar initial speed, speed is gradually lost over time: the shape of $n(x, t)$ at different times for this case is shown in Figure 2(b). We clearly see that no true travelling wave forms, with many agents being left far behind the wave front, leading to its slowing down. Thus, we can interpret growth and death terms in terms of a stabilising role on the wave profile: although not all agents can keep up with the wave, new agents are constantly created at the front and the agents that drop out eventually die, resulting in a travelling band of agents.

\section{From hybrid models to macroscopic PDEs}

In this section we derive macroscopic PDEs for the spatio-temporal density of bacteria $n(x, t)$ at given position $x \in \mathbb{R}$ and time $t \geq 0$. An implicit assumption of the derivation is spatial independence of bacteria, which allows formulation 
(a)

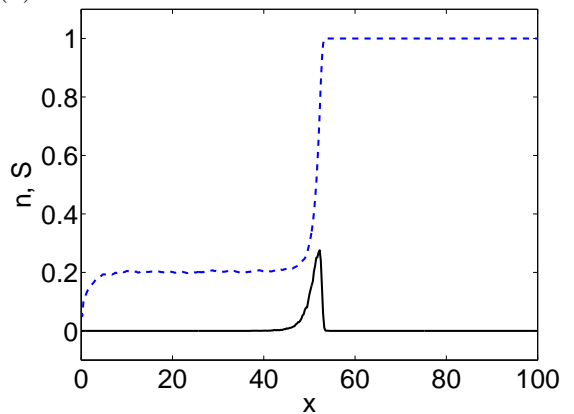

(c)

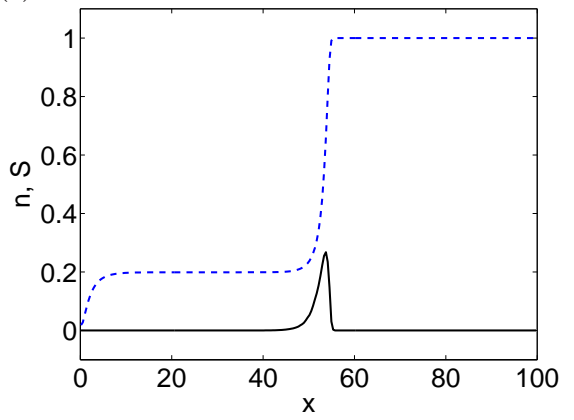

(b)

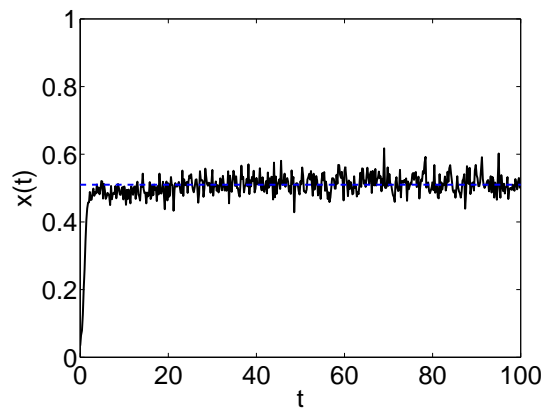

(d)

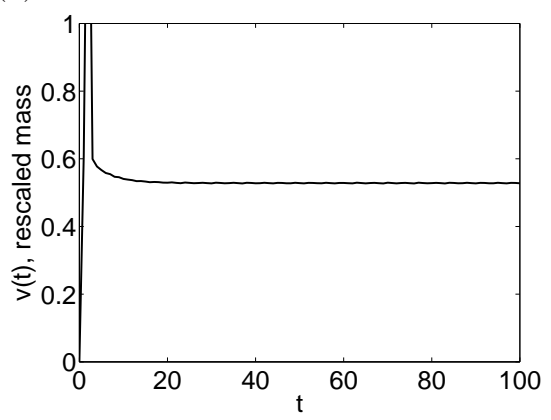

Fig. 1 Numerical solutions of the hybrid chemotaxis model (2.2)-2.6) and 2.10) and PDE System A 3.1 -3.3.

(a) Wave form for the hybrid model after time $t=100$. Solid line: estimated density of bacteria, dashed line: extracellular chemical signal $S$.

(b) Measured speed of travelling wave (solid line). Dashed line denotes the average speed. (c) Wave form for PDE system A after time $t=100$. Solid line: estimated density of bacteria, dashed line: extracellular chemical signal $S$.

(d) Measured speed of travelling wave (solid line) for PDE System A. Note that the spike near $t=0$ is a product of the wave speed calculation method.

The dimensionless parameters are: $\alpha=\beta=s=1, S_{c}=0.5, S_{\infty}=1, \Delta t=10^{-3}$, $\Delta x=0.25, L=100, \lambda_{0}=10, \kappa=0.01, D_{S}=0, t_{a}=0.1, \sigma=0.5$.

of a continuous mesoscopic system. We then use results from 12 to obtain the macroscopic equations. To illustrate the successive formulation of models we construct two systems of PDEs - denoted System (A) and System (B) to be referred to in the remainder of the paper.

\subsection{System (A)}

We define the mesoscopic densities $p^{ \pm}\left(x, y^{(2)}, t\right)$ for left and right-moving bacteria, depending on their position $x \in \mathbb{R}$, their internal variable $y^{(2)} \in \mathbb{R}$ and $t \geq 0$. If the signal profile $S \equiv S(x, t)$ was uninfluenced by bacteria, densities 
(a)

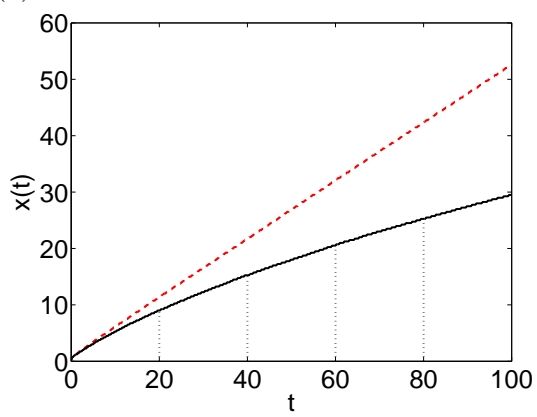

(b)

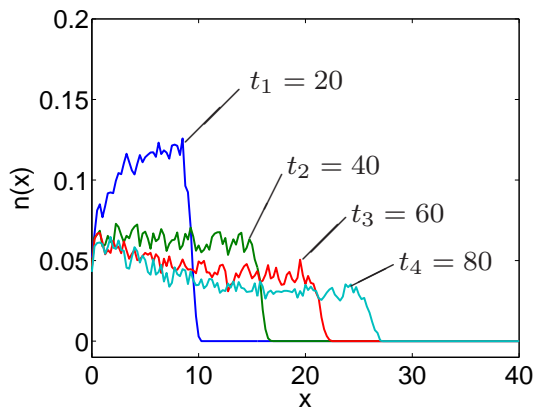

Fig. 2 Numerical solutions of the hybrid chemotaxis model (2.2)-(2.6) and 2.10) without growth and death terms.

(a) Comparison of position of wave front over time. Solid line: without growth/death ( $\alpha=$ $0)$, dashed line: with growth/death $(\alpha=1)$.

(b) Wave form at different times during simulation with $\alpha=0$. From left to right: $t=$ $20,40,60,80$.

Remaining parameters as in Figure 1.

$p^{ \pm}$would satisfy the following system of hyperbolic PDEs:

$$
\begin{aligned}
& \frac{\partial p^{+}}{\partial t}+s \frac{\partial p^{+}}{\partial x}+\frac{\partial}{\partial y^{(2)}}\left(\frac{S(x, t)-y^{(2)}}{t_{a}} p^{+}\right)=-\lambda p^{+}+\lambda p^{-}+h(S(x, t)) p^{+}, \\
& \frac{\partial p^{-}}{\partial t}-s \frac{\partial p^{-}}{\partial x}+\frac{\partial}{\partial y^{(2)}}\left(\frac{S(x, t)-y^{(2)}}{t_{a}} p^{-}\right)=\lambda p^{+}-\lambda p^{-}+h(S(x, t)) p^{-}
\end{aligned}
$$

where $\lambda$ is defined in (2.4) which, under (2.11), can be simplified to

$$
\lambda=\lambda_{0}\left(1-\frac{S(x)-y^{(2)}}{\kappa+\left|S(x)-y^{(2)}\right|}\right) .
$$

The signal dynamics is described by (2.8) which can be rewritten in terms of $p^{ \pm}$as

$$
\frac{\partial S}{\partial t}=D_{S} \frac{\partial^{2} S}{\partial x^{2}}-k(S) \int_{\mathbb{R}}\left(p^{+}+p^{-}\right) \mathrm{d} y^{(2)} .
$$

We denote the system of equations (3.1)-3.3 as System (A).

The system (3.1) (for the one-particle distribution) can be derived by integrating the probability distribution function $p\left(x_{1}, v_{1}, \mathbf{y}_{1} ; x_{2}, v_{2}, \mathbf{y}_{2} ; \ldots \mid S(x, t)\right)$ for the many particle system, utilizing the fact that the movement of individuals are biased by the signal function $S(x, t)$, but independent to each other. However, for the hybrid chemotaxis models described in Sections 2.1 and 2.2 individual bacteria interact via the extracellular signal $S$ which complicates the derivation of (3.1). In 11, a kinetic description has been derived for a model of interacting locusts, using a modified version of the BBGKY hierarchy from the classical kinetic theory of gases [8]. The system we consider here is much more complicated to analyse than the locust model studied in [1], due to the variable number of bacteria and internal variables. Thus the 
kinetic description (3.1) can only be considered as an approximation to the one particle distributions of the interacting system.

The capacity of the above mesoscopic system to generate travelling bands analogous to those observed in the hybrid model is illustrated in Figure 1(c)(d). For details of the numerical method employed for this and other simulations of the continuous model, we refer to [40. The qualitatively and quantitatively close correspondence in solutions under equivalent parameters and initial conditions corroborates the use of the above approximation.

\subsection{System $(\mathbf{B})$}

We consider a macroscopic model in this section. Define the macroscopic densities

$$
p^{ \pm}(x, t)=\int_{\mathbb{R}} p^{ \pm}\left(x, y^{(2)}, t\right) \mathrm{d} y^{(2)},
$$

and let them satisfy the following system

$$
\begin{aligned}
& \frac{\partial p^{+}}{\partial t}+s \frac{\partial p^{+}}{\partial x}=-\lambda^{+}\left(\frac{\partial S}{\partial x}\right) p^{+}+\lambda^{-}\left(\frac{\partial S}{\partial x}\right) p^{-}+h(S) p^{+} \\
& \frac{\partial p^{-}}{\partial t}-s \frac{\partial p^{-}}{\partial x}=\lambda^{+}\left(\frac{\partial S}{\partial x}\right) p^{+}-\lambda^{-}\left(\frac{\partial S}{\partial x}\right) p^{-}+h(S) p^{-}
\end{aligned}
$$

where the turning rates $\lambda^{ \pm}$are given by

$$
\lambda^{ \pm}=\lambda_{0}\left(1 \mp \chi \frac{\partial S}{\partial x}\right) \quad \text { with } \quad \chi=\frac{s t_{a}}{\kappa \lambda_{0}\left(1+2 \lambda_{0} t_{a}\right)}
$$

Using (3.4), equation (3.3) can be written as

$$
\frac{\partial S}{\partial t}=D_{S} \frac{\partial^{2} S}{\partial x^{2}}-k(S)\left(p^{+}+p^{-}\right)
$$

We will denote (3.5) and (3.7) along with the definition of $\lambda^{ \pm}$in (3.6) as System (B). According to the analysis in [12,41, System (B) is quantitatively consistent with System (A) when the external signal $S(x)$ changes slow enough such that cells are close to their fully adapted state, in which case cell movement is only moderately modified by the signal.

In the rest of the paper, we assume diffusion of extracellular signal to occur on a much slower time scale than the active motion of the bacteria, hence $D_{S}=0$. The number of parameters of the above models can be reduced by setting $s, S_{\infty}, \alpha, \beta$ to one through rescaling. We show this in detail for System (B) as follows. Rescaling the variables $S=\hat{S} S_{\infty}, p^{ \pm}=\hat{p}^{ \pm} \alpha S_{\infty} / \beta$, $t=\hat{t} /\left(\alpha S_{\infty}\right), x=\hat{x} s /\left(\alpha S_{\infty}\right)$ and the parameters $S_{c}=\hat{S}_{c} S_{\infty}, \lambda_{0}=\hat{\lambda}_{0} \alpha S_{\infty}$, 
taking (2.7) and substituting into System (B) we obtain, after dropping hats for notational simplicity,

$$
\begin{aligned}
\frac{\partial p^{+}}{\partial t}+\frac{\partial p^{+}}{\partial x} & =-\lambda^{+}\left(\frac{\partial S}{\partial x}\right) p^{+}+\lambda^{-}\left(\frac{\partial S}{\partial x}\right) p^{-}+\left(S-S_{c}\right) p^{+}, \\
\frac{\partial p^{-}}{\partial t}-\frac{\partial p^{-}}{\partial x} & =\lambda^{+}\left(\frac{\partial S}{\partial x}\right) p^{+}-\lambda^{-}\left(\frac{\partial S}{\partial x}\right) p^{-}+\left(S-S_{c}\right) p^{-}, \\
\frac{\partial S}{\partial t} & =-S\left(p^{+}+p^{-}\right) .
\end{aligned}
$$

We are interested in travelling wave solutions that develop from a pointwise inoculation of cells into a domain containing uniformly distributed nutrient $S$. In this scenario, $p^{ \pm}$(defined as in each system) should form travelling pulses while $S$ forms a travelling front and relevant boundary conditions will be

$$
\begin{aligned}
& p^{ \pm}, \frac{\partial p^{ \pm}}{\partial x}, \frac{\partial S}{\partial x} \rightarrow 0 \quad \text { as } \quad x \rightarrow \pm \infty, \\
& S \rightarrow 1 \quad \text { as } x \rightarrow+\infty \text {, } \\
& S \rightarrow S_{-} \quad \text { as } x \rightarrow-\infty \text {. }
\end{aligned}
$$

Note that $S_{-}$is currently unknown; we determine its value in the travelling wave analysis of Section 4 Since $p^{ \pm}$and $S$ are physical quantities, we search for nonnegative travelling wave solutions, i.e.

$$
p^{ \pm} \geq 0, \quad S \geq 0 .
$$

It is clear that a travelling wave of this form cannot exist for $S_{c} \geq 1$ (extinction of bacteria) or for $S_{c} \leq 0$ (infinite growth) and we will therefore only consider systems that satisfy $S_{c} \in(0,1)$. In the next section we analyse System (B) with respect to travelling wave solutions in order to obtain further insight. To do that, we use the rescaled system (3.8).

\section{Travelling wave analysis}

In this section we first apply the standard travelling wave ansatz to system (3.8) and derive a necessary condition for the existence of non-negative travelling wave solutions. We then reduce the resulting ODE system to two components through a change of variables and utilizing an invariant manifold identified for the problem. Finally we use phase plane methods to analyse the existence and properties of travelling wave solutions.

4.1 A necessary condition for the existence of travelling wave solutions

Let us apply the travelling wave ansatz $p^{ \pm}(x, t)=p^{ \pm}(\xi)=p^{ \pm}(x-c t)$ and $S(x, t)=S(\xi)=S(x-c t)$, where $c$ is the unknown wave speed [29]. System 
(3.8) becomes

$$
\begin{aligned}
(1-c)\left(p^{+}\right)^{\prime} & =-\lambda_{0}\left(1-\chi S^{\prime}\right) p^{+}+\lambda_{0}\left(1+\chi S^{\prime}\right) p^{-}+\left(S-S_{c}\right) p^{+}, \\
-(1+c)\left(p^{-}\right)^{\prime} & =\lambda_{0}\left(1-\chi S^{\prime}\right) p^{+}-\lambda_{0}\left(1+\chi S^{\prime}\right) p^{-}+\left(S-S_{c}\right) p^{-}, \\
-c S^{\prime} & =-S\left(p^{+}+p^{-}\right),
\end{aligned}
$$

where the primes denote derivatives with respect to the travelling wave variable $\xi$. Note that any point on the $S$-axis is a steady state of the system (4.1) and that linear stability of such a steady state, $\left(p^{+}, p^{-}, S\right)=\left(0,0, S_{*}\right)$, is governed by the eigenvalues of the matrix $A^{-1} B$, where

$$
A=\left(\begin{array}{ccc}
1-c & 0 & 0 \\
0 & -1-c & 0 \\
0 & 0 & -c
\end{array}\right), \quad B=\left(\begin{array}{ccc}
-\lambda_{0}+S_{*}-S_{c} & \lambda_{0} & 0 \\
\lambda_{0} & -\lambda_{0}+S_{*}-S_{c} & 0 \\
-S_{*} & -S_{*} & 0
\end{array}\right)
$$

The eigenvalues of $A^{-1} B$ are

$$
\mu_{1}=0, \quad \mu_{2,3}=\frac{c\left(-\lambda_{0}+S_{*}-S_{c}\right) \pm \sqrt{\Delta_{1}\left(S_{*}\right)}}{1-c^{2}}
$$

where

$$
\Delta_{1}\left(S_{*}\right)=c^{2} \lambda_{0}^{2}+\left(S_{*}-S_{c}-2 \lambda_{0}\right)\left(S_{*}-S_{c}\right) .
$$

Under the boundary conditions (3.9) we look for nonnegative solutions to (4.1) connecting steady states $\left(p^{+}, p^{-}, S\right)=\left(0,0, S_{-}\right)$and $\left(p^{+}, p^{-}, S\right)=(0,0,1)$. To admit such a solution the latter must be a stable node, since a stable spiral would imply negative values for $p^{ \pm}$. Hence, a necessary condition is $\Delta_{1}(1) \geq 0$, which is equivalent to

$$
c \geq c^{*}=\frac{1}{\lambda_{0}} \sqrt{\left(2 \lambda_{0}-1+S_{c}\right)\left(1-S_{c}\right)} .
$$

Given $2 \lambda_{0}>\left(1-S_{c}\right)$ it is easy to show that $c^{*} \in[0,1]$.

Theorem 1 A necessary condition for the existence of nonnegative travelling wave solutions of the system (3.8) is

$$
2 \lambda_{0}>\left(1-S_{c}\right)
$$

The above condition is reasonable, as we expect the run duration to occur on a much faster time scale than proliferation processes. 
4.2 Dimension reduction

Let us now perform a change of variables by introducing the cell density $n=$ $p^{+}+p^{-}$and the cell flux $j=p^{+}-p^{-}$. The travelling wave system (4.1) can then be written as

$$
\begin{aligned}
-c n^{\prime}+j^{\prime} & =\left(S-S_{c}\right) n, \\
-c j^{\prime}+n^{\prime} & =2 \lambda_{0} \chi S^{\prime} n+\left(S-S_{c}-2 \lambda_{0}\right) j, \\
-c S^{\prime} & =-S n,
\end{aligned}
$$

where the boundary conditions for this system are

$$
\begin{array}{ll}
n, j, \frac{\partial n}{\partial x}, \frac{\partial j}{\partial x}, \frac{\partial S}{\partial x} \rightarrow 0 & \text { as } \quad \xi \rightarrow \pm \infty, \\
S \rightarrow 1 & \text { as } \xi \rightarrow+\infty, \\
S \rightarrow S_{-} & \text {as } \xi \rightarrow-\infty .
\end{array}
$$

From (4.8), we have $S n=c S^{\prime}$ and, hence, $n=c(\ln S)^{\prime}$. Substituting into (4.6) we obtain

$$
-c n^{\prime}+j^{\prime}=c S^{\prime}-c S_{c}(\ln S)^{\prime} .
$$

Integrating and applying the boundary conditions at $\xi \rightarrow+\infty$, an invariant manifold of the problem is given by

$$
-c n+j=c(S-1)-c S_{c} \ln S .
$$

With the definition $f(S) \equiv S-1-S_{c} \ln S$, we obtain $j=c n+c f(S)$, which can be used to eliminate $j$ from the system (4.6)- (4.8). For $c \neq 1$ we can solve for $n^{\prime}$ and obtain the reduced system

$$
\begin{aligned}
n^{\prime} & =\frac{c}{1-c^{2}}\left[\frac{2 \lambda_{0} \chi S n^{2}}{c^{2}}+2 n\left(S-S_{c}-\lambda_{0}\right)+\left(S-S_{c}-2 \lambda_{0}\right) f(S)\right], \\
S^{\prime} & =\frac{1}{c} S n .
\end{aligned}
$$

For $c=1$, we obtain

$$
\begin{aligned}
n & =\frac{\lambda_{0}-S+S_{c}-\sqrt{\left(\lambda_{0}-S+S_{c}\right)^{2}-2 \lambda_{0} \chi S\left(S-S_{c}-2 \lambda_{0}\right) f(S)}}{2 \lambda_{0} \chi S}, \\
S^{\prime} & =S n,
\end{aligned}
$$

where we chose the solution to the quadratic equation for $n$ that satisfies the boundary conditions $n \rightarrow 0$ as $\xi \rightarrow \pm \infty$.

It can be easily shown that $f(S)=0$ has two solutions in the region $(0,1]$ for all $S_{c} \in(0,1)$ as follows. Since $f^{\prime}(S)=1-S_{c} / S, f(S)$ is monotonically decreasing for $S \in\left(0, S_{c}\right)$ and monotonically increasing for $S \in\left(S_{c}, 1\right]$. With $f(1)=0$, this implies $f\left(S_{c}\right)<0$ and, using $f(S) \rightarrow \infty$ for $S \rightarrow 0$, we obtain the existence and uniqueness of the second root of $f(S)=0$ : we call it $S_{1} \in\left(0, S_{c}\right)$. The existence of $S_{1}$ and the negativity of $f(S)$ for $S \in\left(S_{1}, 1\right)$, together with the condition $2 \lambda_{0}>1-S_{c}$, implies that $n$ as given in (4.11) is positive everywhere, and that the given solution therefore satisfies the nonnegativity condition. 
4.3 Steady states and their linear stability

Using the two roots of $f(S)=0$ and under the condition (4.5), it is clear that there are two steady states of the system (4.9)-(4.10): $(n, S)=(0,1)$ and $(n, S)=\left(0, S_{1}\right)$. Linearising the system (4.9)-(4.10) about its steady states generates a system of the form

$$
\left(\begin{array}{l}
n \\
S
\end{array}\right)^{\prime}=A\left(\begin{array}{l}
n \\
S
\end{array}\right),
$$

where, for the general steady state $S_{*} \in\left\{S_{1}, 1\right\}$, we have

$$
A=\left(\begin{array}{cc}
\frac{2 c}{1-c^{2}}\left(S_{*}-S_{c}-\lambda_{0}\right) & \frac{c}{1-c^{2}}\left(S_{*}-S_{c}-2 \lambda_{0}\right) \frac{S_{*}-S_{c}}{S_{*}} \\
\frac{S_{*}}{c} & 0
\end{array}\right)
$$

with

$\operatorname{trace} A=\frac{2 c}{1-c^{2}}\left(S_{*}-S_{c}-\lambda_{0}\right), \quad \operatorname{det} A=-\frac{1}{1-c^{2}}\left(S_{*}-S_{c}-2 \lambda_{0}\right)\left(S_{*}-S_{c}\right)$.

The eigenvalues of $A$ are identical to $\mu_{2,3}$ as given in (4.2). The steady state $(0,1)$ is therefore a stable node for all $c \in\left(c^{*}, 1\right)$ with $c^{*}$ as defined in (4.4). Similarly, it can be seen that the steady steady $\left(0, S_{1}\right)$ is a saddle point. The eigenvectors corresponding to the eigenvalues $\mu_{2,3}$ take the form

$$
\mathbf{v}_{1,2}=\left(\mu_{2,3}, \frac{S_{*}}{c}\right)^{T} .
$$

In the $n-S$ plane, the slopes of the eigenvectors are given by

$$
k_{1,2}(c)=\frac{\mu_{2,3} c}{S_{*}} .
$$

For the steady state $(n, S)=(0,1)$ this slope can be written in the form

$$
k_{1,2}(c)=\frac{c^{2} \lambda_{0}^{2}}{1-S_{c}-\lambda_{0} \mp \sqrt{\Delta}},
$$

where we define $\Delta=c^{2} \lambda_{0}^{2}+\left(1-S_{c}-2 \lambda_{0}\right)\left(1-S_{c}\right)$ similarly to (4.3).

\subsection{Case I: No chemotaxis $(\kappa=\infty)$}

We first consider the case where the chemotactic sensitivity $\chi$ (given by (3.6)) vanishes, i.e cells do not respond chemotactically to changes in $S$. Here, travelling waves are generated solely through proliferation of bacteria at the wave 
(a)

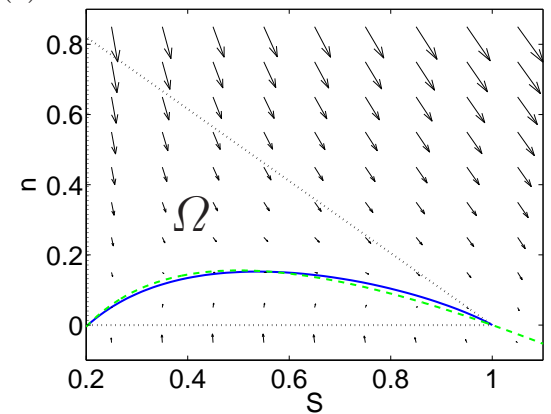

(b)

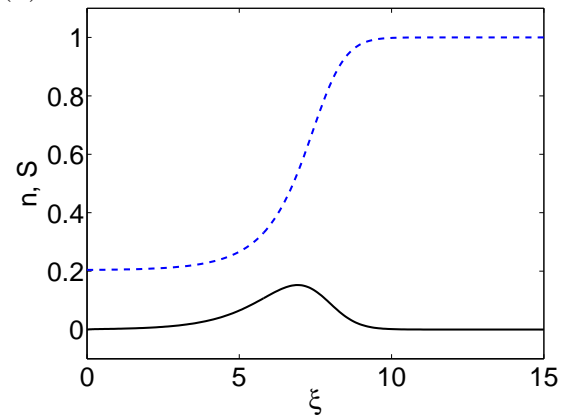

Fig. 3 Illustration of the travelling wave solution calculated using the ODE system (4.9) 4.10) for $\chi=0, \lambda_{0}=10, c=c^{*}=0.3122$ and $S_{c}=0.5$.

(a) Trajectory of travelling wave solution. Solid line: trajectory, dashed line: $n$-nullcline, dotted line: circumference of invariant region $\Omega$ introduced in the proof of Theorem 2

(b) Travelling wave solution in $\xi$. Solid line: $n$, dashed line: $S$.

front. To understand the wave behaviour we perform a phase plane analysis for the ODE system (4.9) - Using $\kappa=\infty$ (i.e. $\chi=0$ ), it reduces to

$$
\begin{aligned}
n^{\prime} & =\frac{c}{1-c^{2}}\left[2 n\left(S-S_{c}-\lambda_{0}\right)+\left(S-S_{c}-2 \lambda_{0}\right) f(S)\right], \\
S^{\prime} & =\frac{1}{c} S n .
\end{aligned}
$$

Thus, the slope of a trajectory in the $n-S$ plane can be written as

$$
\frac{\mathrm{d} n}{\mathrm{~d} S}=\frac{c^{2}}{1-c^{2}} \frac{2 n\left(S-S_{c}-\lambda_{0}\right)+\left(S-S_{c}-2 \lambda_{0}\right) f(S)}{S n} .
$$

Additionally, an expression for the $n$-nullcline $\Gamma_{n}$ is given by

$$
n=-\frac{S-S_{c}-2 \lambda_{0}}{2\left(S-S_{c}-\lambda_{0}\right)} f(S)
$$

and the $S$-nullcline is simply

$$
n=0, \quad \text { or } \quad S=0 .
$$

Let us now show that travelling waves exist for the reduced system (4.15).

Theorem 2 For the case $\chi=0$ (which is equivalent to $\kappa=\infty$ ), a unique travelling wave solution for the system (3.8) exists for all $c \in\left(c^{*}, 1\right)$.

Proof For any $c \in\left(c^{*}, 1\right)$ we can define a region $\Omega$ (see Figure 3(a)), enclosed by the line $n=k_{2}(S-1)$ (with $k_{2}$ defined in (4.14)), the $S$-nullcline $n=0$ and the line $S=S_{1}$. We will first show that $\Omega$ is an invariant region of the system (3.8). Since $S$ is non-decreasing everywhere in $\Omega$ and $n^{\prime}$ is non-negative for $n=0$ and $S \in\left[S_{1}, 1\right]$, we need only to show that the direction field on 
the segment $\Gamma_{1}=\left\{(n, S): n=k_{2}(S-1), S \in\left[S_{1}, 1\right)\right\}$ points from the top half of the plane above this segment towards the bottom. Since $S$ is strictly increasing we require

$$
\left.\frac{\mathrm{d} n}{\mathrm{~d} S}\right|_{\Gamma_{1}} \leq k_{2}(\leq 0) .
$$

Indeed,

$$
\begin{aligned}
\left.\frac{1-c^{2}}{c^{2}} \frac{\mathrm{d} n}{\mathrm{~d} S}\right|_{\Gamma_{1}} & =2 \frac{S-S_{c}-\lambda_{0}}{S}+\frac{\left(S-S_{c}-2 \lambda_{0}\right) f(S)}{S(S-1) k_{2}}, \\
& =2 \frac{S-S_{c}-\lambda_{0}}{S}+\frac{\left(S-S_{c}-2 \lambda_{0}\right) f(S)}{S(S-1) c^{2} \lambda_{0}^{2}}\left(1-S_{c}-\lambda_{0}+\sqrt{\Delta}\right), \\
& \leq 2 \frac{S-S_{c}-\lambda_{0}}{S}+\frac{\left(S-S_{c}-2 \lambda_{0}\right)\left(1-S_{c}\right)}{S c^{2} \lambda_{0}^{2}}\left(1-S_{c}-\lambda_{0}+\sqrt{\Delta}\right),
\end{aligned}
$$

where we used (4.14) in the first step and the relation $f(S) /(S-1) \leq 1-S_{c}$ for all $S \in\left[S_{1}, 1\right]$. Using the fact that $k_{2}$ and $\left(S-S_{c}-2 \lambda_{0}\right)$ are negative, we can use the definition of $c^{*}$ and the fact that $S \leq 1$ to obtain

$$
\begin{aligned}
\left.\frac{1-c^{2}}{c^{2}} \frac{\mathrm{d} n}{\mathrm{~d} S}\right|_{\Gamma_{1}} \leq & 2 \frac{S-S_{c}-\lambda_{0}}{S}-\frac{2 \lambda_{0}+S_{c}-S}{S\left(2 \lambda_{0}+S_{c}-1\right)}\left(1-S_{c}-\lambda_{0}+\sqrt{\Delta}\right), \\
= & \frac{-2 \lambda_{0}^{2}+3 \lambda_{0}\left(S-S_{c}\right)-\left(S-S_{c}\right)\left(1-S_{c}\right)}{S\left(2 \lambda_{0}+S_{c}-1\right)} \\
& -\frac{2 \lambda_{0}+S_{c}-S}{S\left(2 \lambda_{0}+S_{c}-1\right)} \sqrt{\Delta} \\
\leq & \frac{-2 \lambda_{0}^{2}+2 \lambda_{0}\left(S-S_{c}\right)+\lambda_{0}\left(1-S_{c}\right)-\left(S-S_{c}\right)\left(1-S_{c}\right)}{S\left(2 \lambda_{0}+S_{c}-1\right)} \\
& -\frac{2 \lambda_{0}+S_{c}-S}{2 \lambda_{0}+S_{c}-1} \sqrt{\Delta}, \\
= & -\frac{\left(2 \lambda_{0}+S_{c}-1\right)\left(\lambda_{0}+S_{c}-S\right)}{S\left(2 \lambda_{0}+S_{c}-1\right)}-\frac{2 \lambda_{0}+S_{c}-S}{2 \lambda_{0}+S_{c}-1} \sqrt{\Delta} \\
\leq & -\lambda_{0}+1-S_{c}-\sqrt{\Delta}=\frac{1-c^{2}}{c^{2}} k_{2},
\end{aligned}
$$

where we used $S \leq 1$ throughout the derivation. We can therefore conclude that $\Omega$ is an invariant region of the system (3.8). Noting that at the steady state $(n, S)=\left(0, S_{1}\right)$ the unstable manifold has a positive slope $\left(k_{1,2}=\mu_{2,3} c / S_{*}\right)$, i.e. it points into the region $\Omega$, and using the fact that $S$ is strictly increasing inside $\Omega$ for $n>0$ we can conclude that, for each $c \geq c^{*}$, there is a heteroclinic orbit starting from $\left(0, S_{1}\right)$ and finishing at $(0,1)$, corresponding to a travelling wave solution of the PDE system (3.8). 
4.5 Case II: Increasing chemotaxis $(0<\kappa<\infty)$

Decreasing $\kappa$ corresponds to an increase in the chemotactic sensitivity $\chi$ in the ODE system (4.9) (4.10) and the slope of trajectories in the $n-S$ plane is determined by

$$
\frac{\mathrm{d} n}{\mathrm{~d} S}=\frac{c^{2}}{1-c^{2}} \frac{2 n\left(S-S_{c}-\lambda_{0}\right)+\left(S-S_{c}-2 \lambda_{0}\right) f(S)}{S n}+\frac{2 \lambda_{0} \chi}{1-c^{2}} n .
$$

It is noted that the above slope is larger than that for the non-chemotaxis case within the region of interest $n>0$. Due to this increase the region $\Omega$ for the proof of Theorem 1 is no longer invariant for this system and a travelling wave solution to (3.8) does not necessarily exist for all $c \in\left(c^{*}, 1\right)$. The $n$-nullcline for the full ODE system (4.9)-(4.10) is given as the solution of the quadratic equation

$$
\frac{2 \lambda_{0} \chi S}{c} n^{2}+2 c\left(S-S_{c}-\lambda_{0}\right) n+c\left(S-S_{c}-2 \lambda_{0}\right) f(S)=0 .
$$

For a given wave speed $c$, the $n$-nullcline can therefore be calculated as

$$
n=\frac{c}{2 \lambda_{0} \chi S}\left[c\left(\lambda_{0}+S_{c}-S\right) \pm \sqrt{\Delta_{2}(S)}\right]
$$

with

$$
\Delta_{2}(S)=c^{2}\left(\lambda_{0}+S_{c}-S\right)^{2}-2 \lambda_{0} \chi S\left(S-S_{c}-2 \lambda_{0}\right) f(S) .
$$

We can see that $\Delta_{2}(S) \rightarrow-\infty$ as $S \rightarrow \infty$ due to its leading order term $-2 \lambda_{0} \chi S^{3}$. Therefore, as $S$ becomes large, no $n$-nullcline exists and $n^{\prime}$ is positive everywhere. Additionally, $\Delta_{2}(S)$ might have further roots and, in particular, $\Delta_{2}(S)$ might be negative in parts (or the whole) of region $S \in\left[S_{1}, 1\right]$. This again means that $n$ is strictly growing in these parts of the domain.

We detect three different types of behaviours of trajectories starting close to $(n, S)=\left(0, S_{1}\right)$, plotted in Figure 4 In particular, we can see each of these behavioural types for different values of $\chi$ and despite different configurations of the nullclines. In the top two plots of Figure 4 we present the case of a diverging solution. Examining ODE (4.9), we observe that for large $n, n$ grows quicker than $\mathcal{O}\left(n^{2}\right)$ and the divergence can be identified as a finite-time blowup. In the second case, depicted in the two plots in the middle of Figure 4 the trajectory converges to the steady state $(0,1)$, but does so after entering the region $S>1$ and thereafter the region $n<0$. Note that the steady state $(0,1)$ is still a stable node in this case and that this overshoot is therefore not a spiralling effect. Since these trajectories do not correspond to a non-negative solution of the ODE system (4.9)-(4.10), they do not represent travelling wave solutions to the original problem. The last case, presented in the plots on the bottom of Figure 4 corresponds to an acceptable solution and is characterised by the convergence to $(0,1)$ without crossing the line $S=1$. 

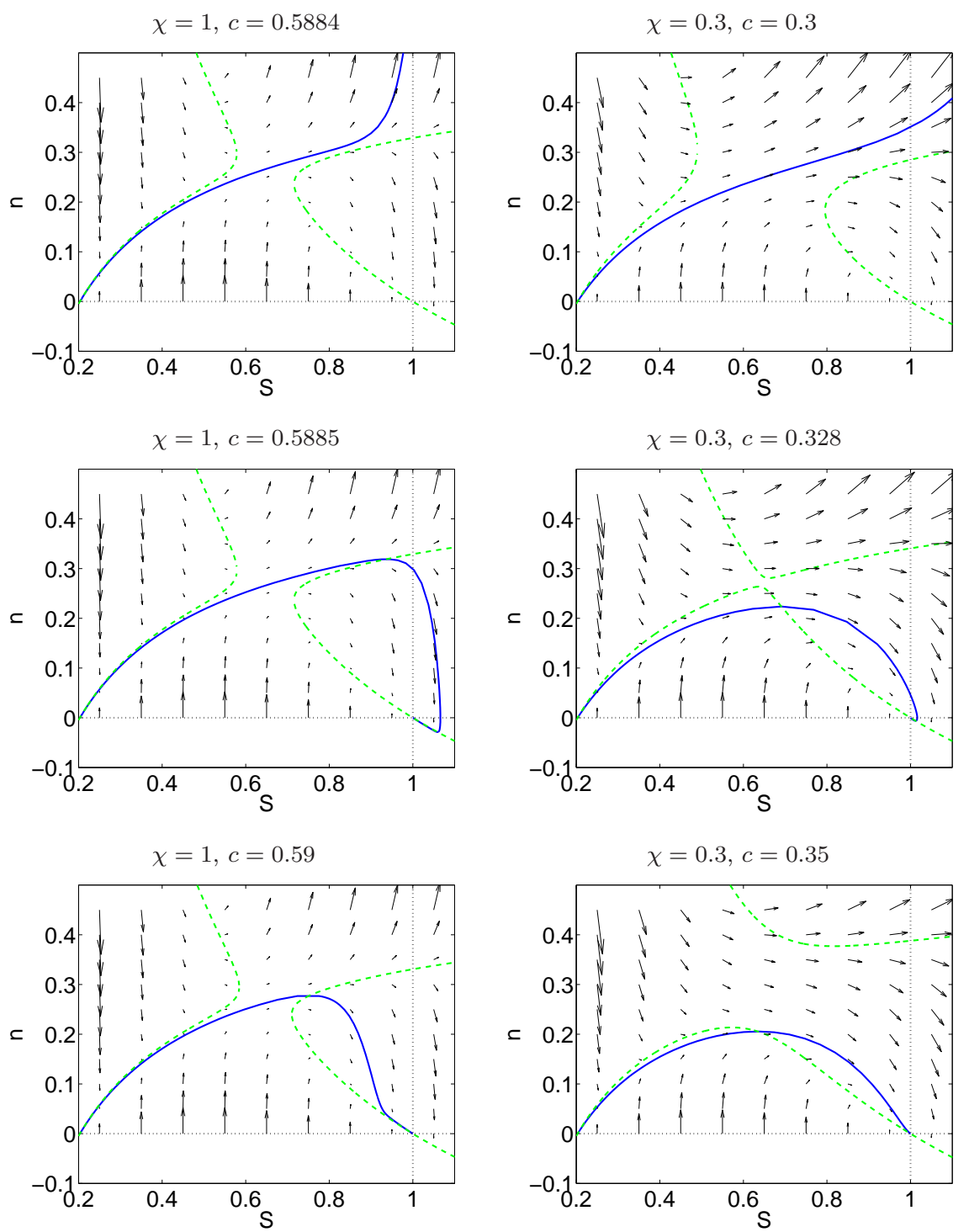

Fig. 4 Trajectories of the ODE system 4.9-4.10 that highlight the three different cases. Parameters in all plots are $\lambda_{0}=10, S_{c}=0.5$. Solid line: trajectory, dashed line: $n$-nullcline, dotted lines: $n=0$ and $S=1$.

4.6 Case III: Infinite chemotactic sensitivity $(\kappa=0)$

As $\kappa$ decreases further we observe that the minimal wave speed necessary to allow a non-negative travelling wave solution of (3.8) increases. In the limit $\kappa \rightarrow 0$, the ODE system (4.9) (4.10) no longer has convergent solutions. However, in this limit the linearisation assumption leading to these ODEs and the 
system (3.8) is no longer valid and we must consider the original turning kernel as defined in (3.2). In the limit $\kappa \rightarrow 0$ the turning rate in the hybrid model therefore becomes

$$
\lambda=\left\{\begin{aligned}
0, & \text { for } y^{(1)}>0, \\
2 \lambda_{0}, & \text { for } y^{(1)}<0 .
\end{aligned}\right.
$$

Hence, bacteria moving in a favourable direction do not turn, indicating that the wave speed achieved in this limit should evolve to $c=s=1$. In 40 it was shown, for a slightly different turning kernel, that travelling waves can exist even without growth terms and that their wave speed satisfies $c=s$.

\section{Computational analysis of the wave speed}

In this section we computationally compare wave speeds from the hybrid model with those of the fully continuous models. Specifically, we investigate the regimes in which the latter provide an acceptable insight into the travelling wave behaviour of the hybrid model, and where they differ. We begin by investigating the non-chemotaxis case, where the minimum wave speed $c^{*}$ for the continuum systems was determined in (4.4). In Section 5.2 we show how the wave speed depends on the value of $\kappa$, and correspondingly the chemotactic sensitivity $\chi$ in the macroscopic model. A comparison with hybrid models without cell proliferation is given in Section 2.3. We conclude this section with a discussion into the effect and origin of oscillations observed under increasing the adaptation time $t_{a}$.

\subsection{Case I: No chemotaxis $(\kappa=\infty)$}

In Section 4.4 we analysed the macroscopic PDEs in the absence of chemotaxis. Travelling wave solutions were shown to exist for all wave speeds $c \in\left(c^{*}, 1\right)$, with $c^{*}$ determined by (4.4). In Figure 5(a), variation of (4.4) as a function of $\lambda_{0}$ is illustrated; we note that wave speeds determined through simulation of the PDE systems correspond exactly (to accuracy of the numerical approximation) with the analytical wave speeds. We now numerically investigate the wave speed for the case $\chi=0$ in the hybrid model.

For our simulations we consider the same parameters and methods as described in Section 2.3 specifically, we set the system parameters $S_{c}=0.5$, $s=1$ and $D_{S}=0$. For the computations we consider a time step $\Delta t=10^{-3}$, a spatial resolution of $\Delta x=0.25$ on a domain with length $L=100$, and simulate the system until the value of $S$ at $x=60$ falls below 0.5 . The profiles at this time, together with the time when $S$ at $x=20$ falls below 0.5 , are used to estimate the wave speed.

The measured wave speed for varying $\lambda_{0}$ is illustrated in Figure 5(a), along with $c^{*}$ as predicted from the travelling wave analysis. While the relationship is similar in shape, we note that at all values of $\lambda_{0}$ tested the measured wave speed lies below the analytical value $c^{*}$. In the literature it has been observed 
(a)

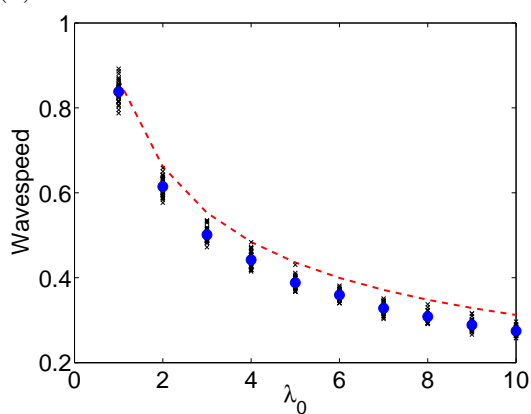

(b)

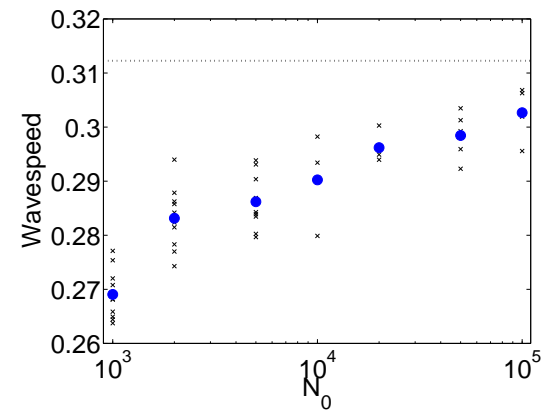

Fig. 5 Measured wave speed in the hybrid model. Crosses: individual simulations, dots: ensemble averages. Parameters are as described in the text.

(a) Wave speed in dependence of $\lambda_{0}$ for $N_{0}=10,000$. Dashed line: $c^{*}$ given by (4.4).

(b) Wave speed as a function of $N_{0}$ with $\lambda_{0}=10$. Dashed line: $c^{*}$ computed by (4.4)

that inaccuracies in numerical schemes can lead to an increase in wave speeds [33, therefore rendering the lower wave speed seen in Figure 5)(a) as counter intuitive.

Nevertheless, we can provide the following explanation for the distinct values in the continuum and hybrid models. For the zero-chemotaxis case, wave generation and movement is solely determined by growth ahead and death behind the wave. In the continuum model an outermost "fractional bacteria population" can extend significantly beyond the wave front, since some proportion of the initial population never turns left, and hence far into the region where $S$ is very close to its initial value of 1 . Yet this fractional population still grows exponentially $\left(\partial p^{ \pm} / \partial t \approx\left(1-S_{c}\right) p^{ \pm}\right)$, seeding the growth and expansion of the population. The finite/discrete nature of the hybrid model precludes any fractional bacterium: the forward "tail" is necessarily finite and growth will not occur beyond the outermost individual.

For the above explanation to hold we would expect a dependence of the measured wave speed on the initial number of bacteria $N_{0}$ : continuous densities provide a closer approximation under larger numbers of bacteria and we would expect convergence in the wave speed to $c^{*}$. Simulations in Figure 5 (b) demonstrate this property, corroborating our interpretation.

\subsection{Case II: Increasing chemotaxis $(0<\kappa<\infty)$}

In the second set of numerical experiments we measure the dependency of the wave speed on the critical parameter $\kappa$, i.e. we determine the effect of increasing chemotaxis as $\kappa$ decreases. We compare the results measured for the hybrid system with the continuous Systems (A) and (B).

We use the same parameters as in Section 5.1 and results are shown in Figure 6. The results demonstrate the regimes where correspondence across the varying modelling levels occurs: while the hybrid model (dotted line) cor- 


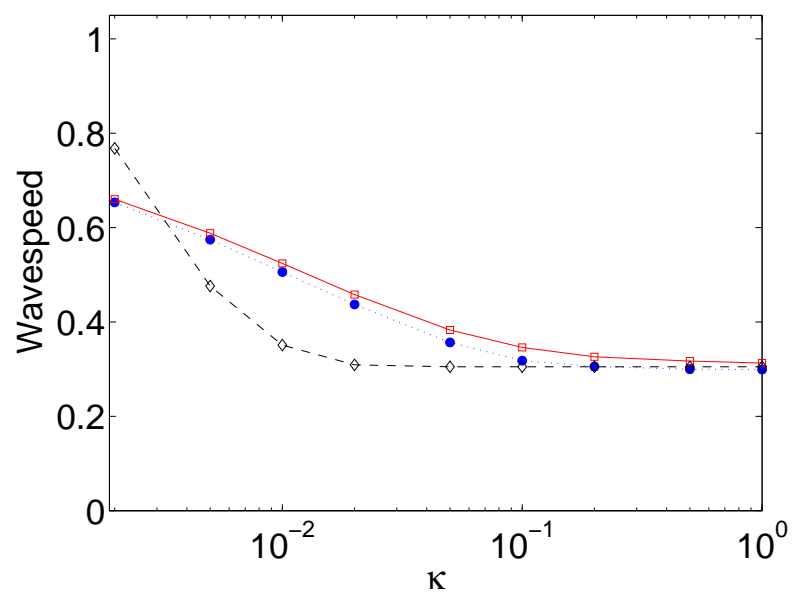

Fig. 6 Comparison between wave speeds of the various models in dependence of $\kappa$. Dotted line: hybrid model, red solid line: mesoscopic System $(\boldsymbol{A})$, dashed line: linearised System (B). Parameters are as described in the text.

responds well with its closest continuous version (mesoscopic System (A), red solid line) over a wide range of $\kappa$, it only corresponds with System (B) (black dashed line) for larger $\kappa$, diverging as $\kappa$ decreases. Note that the turning rate (3.6) used for System (B) becomes negative at small values of $\kappa$ and we limit the range of $\kappa$ studied accordingly.

At larger $\kappa$ all three models converge to a value close to $c^{*}$ as $\kappa$ grows: in this regime the main assumption proposed for the linearisation $\left(\left|S(x)-y^{(2)}\right| \ll\right.$ $\kappa)$ holds and we obtain good quantitative agreement. While this assumption becomes less acceptable as we decrease $\kappa$, leading to the divergent behaviour described above, we note that all models show the same qualitative agreement: increasing chemotactic responses leads to an increase in the wave speed. Note that the results for System (B) can be identically replicated using the ODE system (4.9) -4.10) and a search algorithm for the smallest value of $c$ that admits a nonnegative solution to the system.

These numerical experiments demonstrate that chemotaxis has a significant effect on the speed of movement and that the waves cannot solely be explained by growth and death terms. Rather, we interpret birth and death processes as stabilisers to what would otherwise be transient waves 15,40. This interpretation is in agreement with the results presented in Figure 2 as the initial wave speed for the system without growth seems to be similar to the wave speed of the system including growth and death terms.

\subsection{Oscillations in the wave speed}

An additional observation we made during the numerical experiments of the hybrid model is that for increasing values of the adaptation time $t_{a}$, the wave 
speed starts to differ strongly from the mesoscopic System (A), an effect that we identified to be due to oscillations in the behaviour of the wave. In Figure 7(a) we present an example of strongly oscillating wave speeds (where the wave speed is measured as rate of change of the average position of bacteria). This example occurred for the parameters $S_{c}=0.5, \lambda_{0}=10, \kappa=0.001$ and $t_{a}=4$. We can also clearly see that the wave speed is correlated to the current number of agents in the system. In the literature similar effects of oscillating waves in stochastic models have been observed [28, 32 .

In Figure 7(b), we present the form of the wave at different times throughout the simulation. It is clearly visible that the shape differs significantly at different times. One reason these oscillations occur when $t_{a}$ is very high is that a bacterium that happens to be in front of the wave experiences a very high value of $S$, whilst its internal dynamics only adapt very slowly. This, in combination with the low value of $\kappa$, leads to a bacterium that does not switch direction for a long time and will proliferate at a high rate. This implies that a spike of bacteria forms in front of the wave that moves faster than the rest of the wave. We can clearly see such a spike in the left-most waveform in Figure 7(b). Once the frontrunning bacterium and its copies have turned, the wave goes into a reordering phase (second and third waveform), until, eventually, a new spike emerges (4th waveform).

In Figure 7 (c) we plot the wave speed over time for a smaller value of $t_{a}$. We can see that the oscillations are less severe and more frequent than in Figure 7(a), which is in agreement with the explanation above. As we decrease $t_{a}$ the frontrunning bacteria will adapt quicker to their surroundings and are thereby more likely to turn. We show the influence of changing $N_{0}$ on the oscillating behaviour in Figure $7(\mathrm{~d})$. The oscillations seem to occur with a similar frequency but more regular to those before, which can be explained by the increased likelihood of frontrunning bacteria with a higher number of agents and reduced noise in the system.

\section{Discussion}

In this paper we presented a hybrid model of chemotaxis, incorporating a biologically realistic turning kernel introduced in [40. We analysed the travelling wave behaviour of this hybrid system using mesoscopic and macroscopic equations, deriving an analytical value for the expected wave speed in the case of no chemotaxis. As chemotaxis increases we demonstrated (analytically and numerically) that the expected wave speed increases, indicating that the wave that forms is not solely driven by growth and death processes. In contrast to the transient waves observed for the hybrid model in the absence of growth and death terms [15, the (numerical) waves observed here in their presence are stable, indicating the stabilising effect of birth and death. The numerical analysis reveals that the macroscopic equations derived through linearisation of the turning kernel can qualitatively describe the change in wave speed as chemotaxis increases, but that there are significant quantitative differences 
(a)

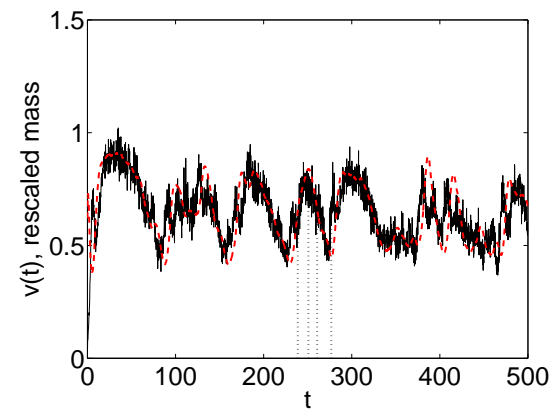

(c)

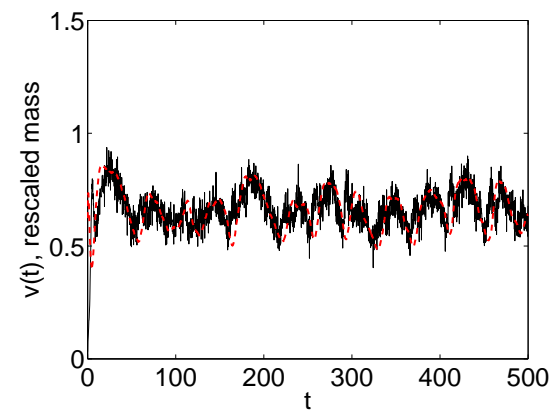

(b)

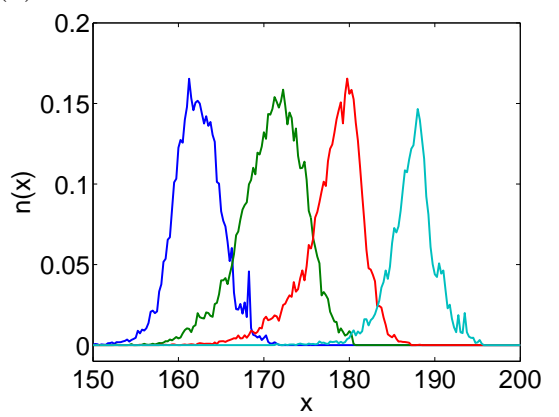

(d)

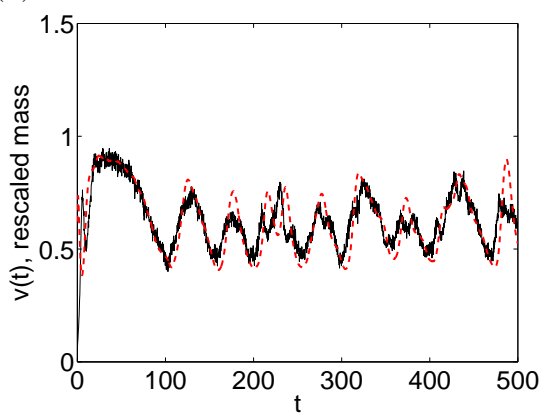

Fig. 7 Oscillations in the wave speed of the hybrid model (2.2)-2.6) and (2.10).

(a) Wave speed in comparison to current number of particles for $N_{0}=10,000, t_{a}=4$. Solid line: wave speed, dashed line: number of particles, dotted lines: times of wave forms shown in panel (b).

(b) Waveform at 4 distinct times marked in panel (a) from left to right.

(c) As in (a) with $N_{0}=10,000, t_{a}=2$.

(d) As in (a) with $N_{0}=50,000, t_{a}=4$.

Other parameters are given in Section 5.3

between the two systems. Additionally, we observed oscillations in the wave movement, an effect that had been seen in similar systems in the literature 32 and that cannot be explained using mean-field approximations.

To date, travelling waves in chemotaxis models have mainly been analysed from the perspective of macroscopic PDE models of chemotaxis [19,18. The existence of travelling waves for continuum models with growth terms is well established [36, 30,22. While hybrid models have been used to study pattern formation in bacterial chemotaxis [17,39, these studies have not analysed the travelling wave patterns observed in bacterial cell populations.

Recently, experimental studies using microfluidic techniques tracked cell trajectories within a traveling pulse, and revealed that persistence of direction in cell movement accounts for $30 \%$ of the macroscopic speed of the traveling pulse 35 . The hybrid model framework studied here provides a natural method for direct comparison of model predictions with experimental measurements of cell trajectory, and this is left as future work. 


\section{Acknowledgements}

The research leading to these results has received funding from the European Research Council under the European Community's Seventh Framework Programme (FPr/200\%-2013) / ERC grant agreement No. 239870. This publication was based on work supported in part by Award No KUK-C1-013-04, made by King Abdullah University of Science and Technology (KAUST). Radek Erban would also like to thank the Royal Society for a University Research Fellowship; Brasenose College, University of Oxford, for a Nicholas Kurti Junior Fellowship; and the Leverhulme Trust for a Philip Leverhulme Prize. This prize money was used to support research visits of Chuan Xue and Kevin Painter in Oxford. Kevin Painter acknowledges a Leverhulme Trust Research Fellowship award (RF-2011-045).

\section{References}

1. J. Adler. Chemotaxis in bacteria. Science, 153:708-716, 1966

2. H. Berg. How bacteria swim. Scientific American, 233:36-44, 1975.

3. H. Berg and D. Brown. Chemotaxis in Esterichia coli analysed by three-dimensional tracking. Nature, 239:500-504, 1972.

4. R. Bourret, K. Borkovich, and M. Simon. Signal transduction pathways involving protein phosphorylation in prokaryotes. Annual Review of Biochemistry, 60:401-441, 1991.

5. M. Brenner, L. Levitov, and E. Budrene. Physical mechanisms for chemotactic pattern formation by bacteria. Biophysical Journal, 74(4):1677-1693, 1998.

6. E. Budrene and H. Berg. Complex patterns formed by motile cells of Esterichia coli. Nature, 349:630-633, February 1991.

7. E. Budrene and H. Berg. Dynamics of formation of symmetrical patterns by chemotactic bacteria. Nature, 376:49-53, July 1995.

8. C. Cercignani, R. Illner, and M. Pulvirenti. The Mathematical Theory of Dilute Gases. Applied Mathematical Sciences, 106, Springer-Verlag, 1994.

9. P. Chavanis. A stochastic Keller-Segel model of chemotaxis. Communications in nonlinear science and numerical simulations, 15:60-70, 2010.

10. R. Erban. From individual to collective behaviour in biological systems. PhD thesis, University of Minnesota, 2005.

11. R. Erban and J. Haskovec. From individual to collective behaviour of coupled velocity jump processes: A locust example. Kinetic and Related Models, 5(4):817-842, 2012.

12. R. Erban and H. Othmer. From individual to collective behaviour in bacterial chemotaxis. SIAM Journal on Applied Mathematics, 65(2):361-391, 2004.

13. R. Erban and H. Othmer. From signal transduction to spatial pattern formation in $E$. coli: A paradigm for multi-scale modeling in biology. Multiscale Modeling and Simulation, 3(2):362-394, 2005.

14. R. Fisher. The wave of advance of advantageous genes. Annals of Eugenics, 7:355-369, 1937.

15. B. Franz and R. Erban. Hybrid modelling of individual movement and collective behaviour. In M. Lewis, P. Maini, and S. Petrovskii, editors, Dispersal, individual movement and spatial ecology: A mathematical perspective. Springer, 2013.

16. A. Gerisch and K. Painter. Mathematical modelling of cell adhesion and its applications to developmental biology and cancer invasion. In A. Chauviere and L. Preziosi, editors, Cell Mechanics: From Single Scale-Based Models to Multiscale Modeling, Chapter 12 pages 319-350. CRC Press, 2010.

17. Z. Guo, P. Sloot, and J. Tay. A hybrid agent-based approach for modeling microbiological systems. Journal of Theoretical Biology, 255:163-175, 2008.

18. T. Hillen and K. Painter. A user's guide to pde models for chemotaxis. Journal of Mathematical Biology, 58:183-217, 2009. 
19. D. Horstmann. From 1970 until present: the Keller-Segel model in chemotaxis and its consequences II. Jahresbericht des Deutschen Mathematiker Vereins, 106:51-69, 2004.

20. E. Keller and L. Segel. Traveling bands of chemotactic bacteria: A theoretical analysis. Journal of Theoretical Biology, 30:235-248, 1971.

21. C. Kennedy and R. Aris. Traveling waves in a simple population model involving growth and death. Bulletin of Mathematical Biology, 42:397-429, 1980.

22. K. Landman, G. Petter, and D. Newgreen. Chemotactic cellular migration: smooth and discontinuous travelling wave solutions. SIAM Journal on Applied Mathematics, 63(5):1666-1681, 2003.

23. K. Landman, M. Simpson, J. Slater, and D. Newgreen. Diffusive and chemotactic cellular migration: Smooth and discontinuous travelling wave solutions. SIAM Journal of Applied Mathematics, 65:1420-1442, 2005.

24. K.A. Landman, M.J. Simpson, and D.F. Newgreen. Mathematical and experimental insights into the development of the enteric nervous system and hirschsprung's disease. Development, growth and differentiation, 49:277-286, 2007.

25. T. Li and Z. Wang. Asymptotic nonlinear stability of traveling waves to conservation laws arising from chemotaxis. Journal of Differential Equations, 250:1310-1333, 2011.

26. T. Li and Z. Wang. Steadily propagating waves of a chemotaxis model. Mathematical Biosciences, 240:161-168, 2012.

27. R. Lui and Z. Wang. Traveling wave solutions from microscopic to macroscopic chemotaxis models. Journal of Mathematical Biology, 61:739-761, 2010.

28. M. Metcalf, J. Merkin, and S. Scott. Oscillating wave fronts in isothermal chemical systems with arbitrary powers of autocatalysis. Proceedings of the Royal Society London A, 447:155-174, 1994.

29. J. Murray. Mathematical Biology. Springer Verlag, 2002.

30. G. Nadin, B. Perthame, and L. Ryzhik. Traveling waves for the Keller-Segel system with Fisher birth term. Interfaces and Free Boundaries, 10:517-538, 2008.

31. H. Othmer, S. Dunbar, and W Alt. Models of dispersal in biological systems. Journal of Mathematical Biology, 26:263-298, 1988.

32. H. Othmer and P. Schaap. Oscillatory cAMP signaling in the development of Dictyostelium discoideum. Comments on Theoretical Biology, 5:175-282, 1998.

33. R.D. Reitz. A study of numerical methods for reaction-diffusion equations. SIAM Journal on Scientific and Statistical Computing, 2:95-106, 1981.

34. J. Saragosti, V. Calvez, N. Bournaveas, A. Buguin, P. Silberzan, and B. Perthame. Mathematical description of bacterial traveling pulses. PLoS Computational Biology, 6:e1000890, 2010

35. J. Saragosti, V. Calvez, N. Bournaveas, B. Perthame, A. Buguin, and P. Silberzan. Directional persistence of chemotactic bacteria in a traveling concentration wave. Proceedings of the National Academy of Sciences, 108(39):16235-16240, 2011.

36. R. Satnoianu, P. Maini, F. Garduno, and J. Armitage. Travelling waves in a nonlinear degenerate diffusion model for bacterial pattern formation. Discrete and Continuous Dynamical Systems B, 1:339-362, 2001.

37. Z.A. Wang. Mathematics of traveling waves in chemotaxis - review paper. Discrete and Continuous Dynamical Systems Series B, 13:601-641, 2013.

38. M. B. Witte and A. Barbul. General principles of wound healing. Surgical Clinics of North America, 77:509-528, 1997.

39. C. Xue, E. Budrene, and H. Othmer. Radial and spiral streams in proteus mirabilis colonies. PLoS Computational Biology, 7(12):e1002332, 2011.

40. C. Xue, H. Hwang, K. Painter, and R. Erban. Travelling waves in hyperbolic chemotaxis equations. Bulletin of Mathematical Biology, 73(8):1695-1733, 2011.

41. C. Xue and H. Othmer. Multiscale models of taxis-driven patterning in bacterial populations. SIAM Journal on Applied Mathematics, 70(1):133-167, 2009. 